\title{
PENTASTOMES DE REPTILES MALGACHES
}

\author{
Par Simon GRETILLAT, Edouard-R. BRYGOO et Charles-A. DOMERGUE
}

La faune des Pentastomes parasites de l'appareil pulmonaire des Reptiles à Madagascar est à notre connaissance encore très mal connue.

Quatre espèces seulement ont été signalées ou décrites de la Grande Ile. Ce sont :

Gigliolella brumpti (Giglioli, 1922) Chabaud et Choquet, 1954 (= Armillifer brumpti Giglioli, 1922), dont les formes adultes avaient été recueillies par M. A. Mocquérys en 1898, « chez un serpent dont l'espèce et le genre n'ont pas été mentionnés \$ (d'après Giglioli), et les formes nymphales chez un « hérisson 》.

Heymons et Vitzthum (1936) donnent comme hôte de ce Pentastome Boa madagascariensis Duméril et Bribon (nach Giglioli ?) et, d'après leurs propres observations, Corallus madagascariensis (Duméril et Bribon).

$\mathrm{Au}$ sujet de l'origine des formes nymphales signalées par Giglioli, comme il n'y a pas de hérisson à Madagascar, elles n'ont pu être récoltées que chez Setifer (Ericulus) setosus, petit Insectivore malgache qui, par son apparence extérieure, ressemble à un hérisson européen, mais qui n'est pas le Tenrec (Tenrec ecaudatus)...

Kiricephalus pattoni (Stephens, 1908) est signalé par Miss Hett en 1924 chez Lioheteredon madagascariensis Duméril et Bribon, et par Heymons et Vitzthum en 1936 chez Corallus madagascariensis.

Raillietiella chamaeleonis Grétillat et Brygoo, 1959, décrit de Chamaeleo oustaleti Mocquard, 1894 et de Chamaeleo verrucosus Cuvier, 1829.

Raillietiella (Heymonsia) hemidactylis Hett, 1934, signalé pour la première fois à Madagascar chez Hemidactylus frenatus Schlegel, par Grétillat et Brygoo en 1961, et trouvé par nous en octobre 1960 dans les sacs aériens d'un Chamaeleo oustaleti.

C'est grâce à de nombreuses récoltes et autopsies de Reptiles provenant de différentes régions de la Grande Ile, que deux d'entre nous ont pu recueillir le matériel dont les résultats de l'étude font l'objet de cette note.

\section{Gigliolella brumpti (Giglioli, 1922) Chabaud et Choquet, 1954}

Un mâle et une femelle trouvés dans les sacs aériens d'un Acrantophis dumerili Jan, 1860 (Ophidien), à Egogy, district d'Ampanihy (Sud de Madagascar) (R. 94, $\mathrm{n}^{\circ}$ du tube 73/61, le 22-3-1961).

Dans une petite note (Grétillat et Brygoo, 1961), nous avons fait remarquer que la forme et les dimensions des * dilatateurs de copulation 》 des mâles de 


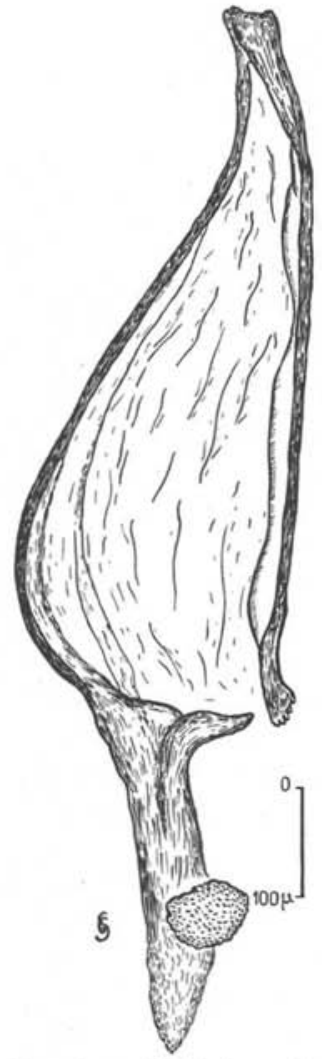

Fig. 1. - Dilatateur de copulation de Gigliolella brumpti (Giglioli, 1922), Chabaud et Choquet, 1954

Pentastomida auraient peut-être une valeur taxonomique dans la diagnose des espèces de ce groupe.

Pour cette raison, nous donnons ci-dessous la description de ces organes chez $G$. brumpti en soulignant que dans cette espèce ils sont complètement différents de ceux déjà décrits chez Raillietiella chamaeleonis et Raillietiella (Heymonsia) hemidactyli.

Chez G. brumpti, les « dilatateurs de copulation » ont la forme suivante : ce sont deux pièces fortement chitinisées (fig. 1), de $1.000 \mu$ de longueur environ, dont la partie antérieure, cylindroïde et pointue, est flanquée latéralement d'un tubercule sensiblement hémisphérique à surface chagrinée. La partie postérieure du dilatateur a environ $700 \mu$ de longueur et a la forme d'un V dont la pointe est dirigée vers l'arrière du Pentastome. Deux branches chitinisées partent de ce sommet. L'externe est à peu près rectiligne. L'interne est convexe et s'unit en avant avec la partie cylindrique du dilatateur. Elles sont reliées entre elles par une membrane qui est un peu plus épaisse le long de la branche interne.

\section{Raillietiella chamaeleonis}

Grétillat et Brygoo, 1959

Un mâle trouvé dans les sacs aériens d'un Lioheterodon modestus (Gunther, 1863) (Ophidien), à Ejeda, Betioky (Sud de Madagascar) (Rec. 63, $\mathrm{n}^{\circ}$ du tube 29/61, 29-4-1961).

Cette espèce ne serait donc pas seulement un parasite de caméléon, mais pourrait aussi parasiter éventuellement des Serpents.

\section{Raillietiella ampanihyensis n. sp.}

Matériel : Deux mâles et cinq femelles trouvés dans les sacs aériens d'un Madagascarophis colubrina (Schlegel, 1837) (Ophidien), à Tongobory (Rec. 65, $\mathrm{n}^{\circ} \mathrm{du}$ tube 34/61, 25-4-61).

Un mâle dans l'appareil pulmonaire d'un Lioheterodon madagascariensis Duméril et Bribon (Ophidien) à Vohitsara (Rec. 90, $\mathrm{n}^{\circ}$ du tube 70/61, 4-4-61). 
Male (fig. 2).

Dimensions et habitus: Les chiffres que nous donnons correspondent à des moyennes obtenues d'après les trois exemplaires que nous avions à notre disposition.

Longueur: 9,5 mm. environ.

Largeur maxima du corps : 1,2 mm. environ, au niveau du tiers antérieur.

Fixé dans l'alcool, ce Pentastome a une coloration jaune, légèrement ambrée dans la partie antérieure.

Le céphalothorax, qui a une forme sensiblement triangulaire, ne présente pas de séparation bien marquée avec l'abdomen qui, légèrement aplati ventralement dans son tiers antérieur, est régulièrement effilé vers l'arrière où il présente deux appendices coniques, charnus, bien développés, mais sans «bouton terminal». Chaque appendice mesure environ $250 \mu$ de long et sa base, qui est ventro-terminale, a une épaisseur de $115 \mu$ (fig. 3).

L'abdomen est segmenté en 40 à 42 anneaux bien marqués présentant chacun une paire de petits renflements pédonculaires latéro-ventraux (fig. 2).

Le tube digestif apparaît par transparence allongé, non circonvolutionné, marron foncé, se terminant

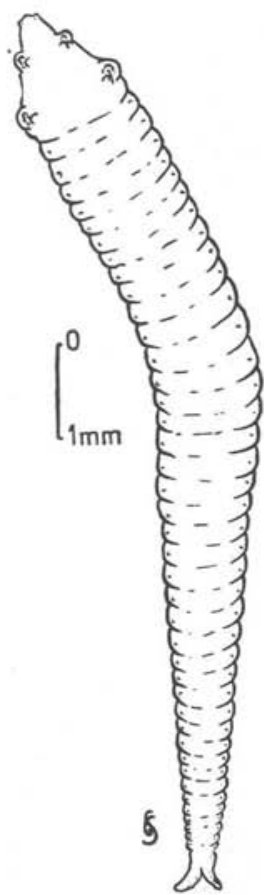

Fig. 2. - Raillietiella ampanihyensis n. sp. (mâle) par un anus ventral au niveau de la partie postérieure de l'abdomen.

Céphalothorax (fig. 4): La tête proprement dite, de forme trapézoïdale, a son bord antérieur légèrement convexe et ses angles antérieurs sont occupés par les papilles dorso-latérales bien développées et les papilles frontales beaucoup plus petites placées en région légèrement ventrale.

Le complexe buccal est cordiforme et n'offre rien de bien particulier, si ce ne sont les caractères généraux rencontrés chez les Raillietiella.

Les quatre crochets sont disposés en trapèze très en arrière de la bouche et sont placés chacun au fond d'une cavité occupant le sommet d'un renflement pédonculaire débordant largement le contour dorsal du céphalothorax.

Les bords ventraux et dorsaux de cette cavité sont bordés chacun par une lèvre semi-circulaire (Lobiparapodiale).

Chaque crochet est flanqué latéralement de deux parapodiales. Il a sa partie antérieure crochue coiffée et engainée dans un onychium formé par une expansion cuticulaire du bord antérieur du renflement pédonculaire supportant le complexe crochet-parapodiales (fig. 5). 
Crochets antérieurs (fig. 6) : Ils mesurent $240 \mu$ de long environ sur $50 \mu$ d'épaisseur maximum. Leur base présente une très courte portion cylindroïde dont l'extrémité sert de point d'insertion aux muscles du crochet. Les parapodiales, dont la base est fortement renflée, sont légèrement incurvées et leur extrémité antérieure est quelque peu flabelliforme. Leur longueur moyenne est d'environ $150 \mu$.

Crochets postérieurs (fig. 7): Ils sont plus puissants que les antérieurs et mesurent $270 \mu$ de long sur $85 \mu$ d'épaisseur maxima. Leur allure et leur forme générale rappellent celles des crochets antérieurs. Il en est de même pour les parapodiales qui ont environ $180 \mu$ de longueur.

Appareil génital : Les deux testicules, qui mesurent $700 \mu$ de long sur $250 \mu$ d'épaisseur, sont oblongs et allongés le long des parois abdominales au niveau des cinq à six premiers segments abdominaux. La vésicule séminale, qui est de grandes dimensions, est placée entre eux deux. Son contenu se déverse dans deux canaux déférents circonvolutionnés présentant un renflement ampoulaire vers le milieu de leur parcours, point vers lequel ils s'accolent chacun à la face interne des " dilatateurs de copulation \$ avant d'aboutir au pénis situé au milieu du pore génital placé ventralement sur le premier segment abdominal.

La forme des « dilatateurs de copulation », qui ont environ $800 \mu$ de long, est tout à fait caractéristique. Ce sont deux organes chitineux en forme de massue dont la tête renflée mesure environ $330 \mu$ de long sur $150 \mu$ d'épaisseur. Ce renflement a sa surface ornementée de crêtes et de dépressions plus ou moins allongées (fig. 8).

Le contour externe de chaque * dilatateur » est très fortement chitinisé et il est bordé dans son tiers antérieur par une expansion alaire. Par contre, le bord interne est mince et de contour plus ou moins distinct, surtout au niveau de sa partie moyenne.

Antérieurement, chacun de ces organes se termine par six petites digitations, dont trois pour chacune des branches.

\section{Femelle :}

\section{Dimensions et habitus (fig. 9).}

Longueur : 2,2 à $2,4 \mathrm{~cm}$. suivant les spécimens.

Largeur maximum (tiers antérieur du corps) : 0,21 à $0,23 \mathrm{~cm}$.

Le corps, qui est à peu près cylindrique, est cependant légèrement aplati ventralement dans son quart antérieur, où il présente sa largeur maximum, pour s'effiler graduellement vers son extrémité postérieure.

L'abdomen, qui ne présente qu'un léger étranglement au niveau de son union avec le céphalothorax, a 60 à 64 anneaux suivant les exemplaires.

Les deux appendices terminaux sont coniques et n'ont pas de renflement distal. Ils sont très développés et mesurent $540 \mu$ de long pour une épaisseur basale de $175 \mu$. Ils ne sont pas franchement terminaux mais plutôt ventro- 


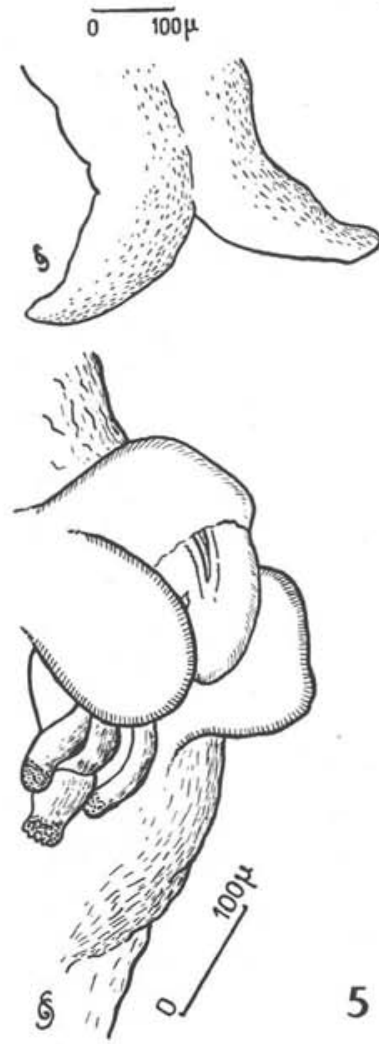

Fig. 3. - Appendices terminaux du mâle de $R$. ampanihyensis

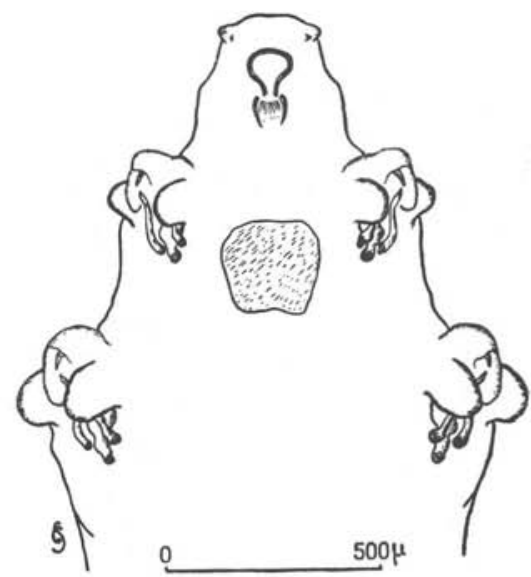

Fig. 4. - Céphalothorax du mâle de $R$. ampanihyensis

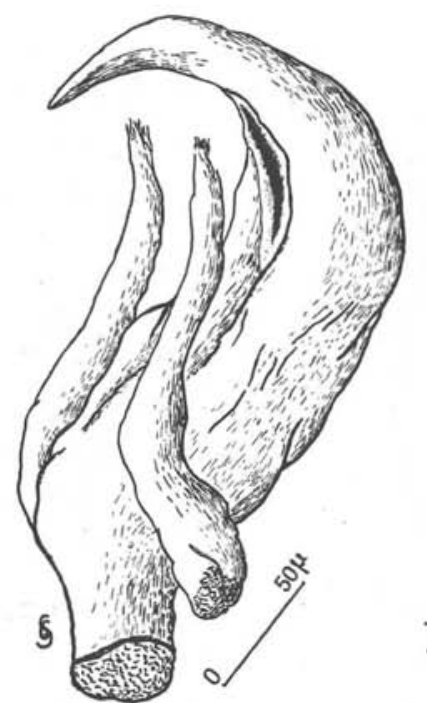

FIG. 5. - R. ampanihyensis. Complexe parapodial (mâle)

Fig. 6. - R. ampanihyensis. Crochet et parapodiales antérieures (mâle)

Fig. 7. - R. ampanihyensis. Crochet et parapodiales postérieures (mâle) 

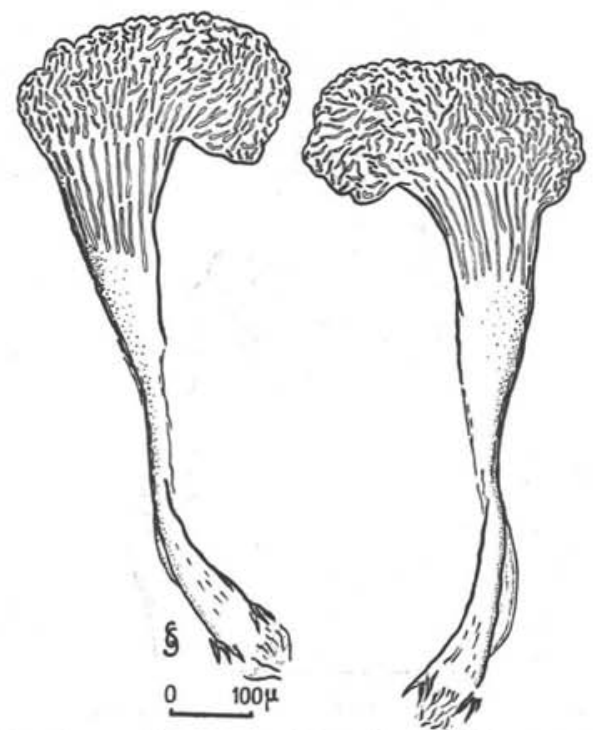

Fig. 8. - R. ampanihyensis. "Dilatateur de copulation

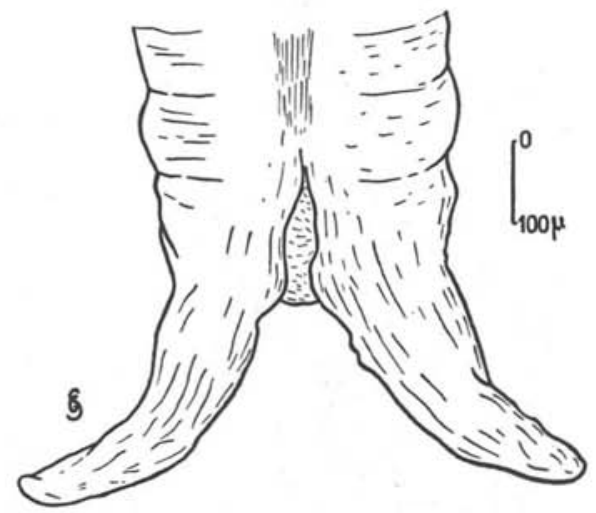

FIG. 10. - R. ampanihyensis, appendices terminaux de la femelle

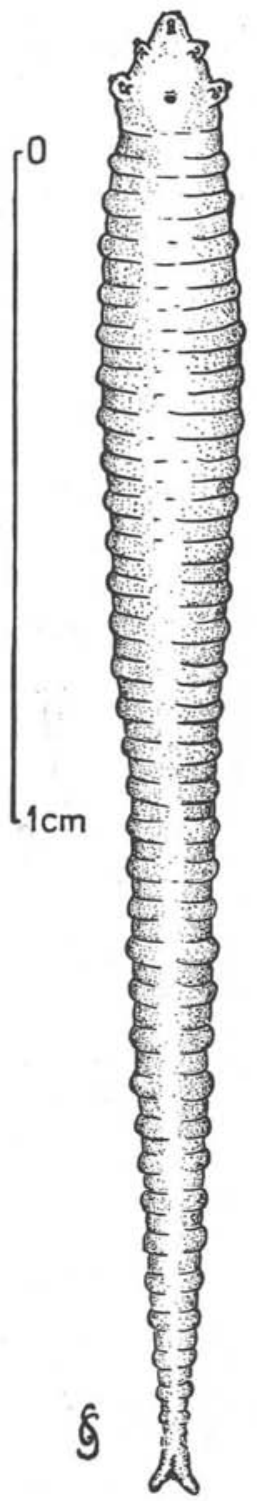

F1G. 9. - Raillietiella ampanihyensis n. sp. (femelle) 


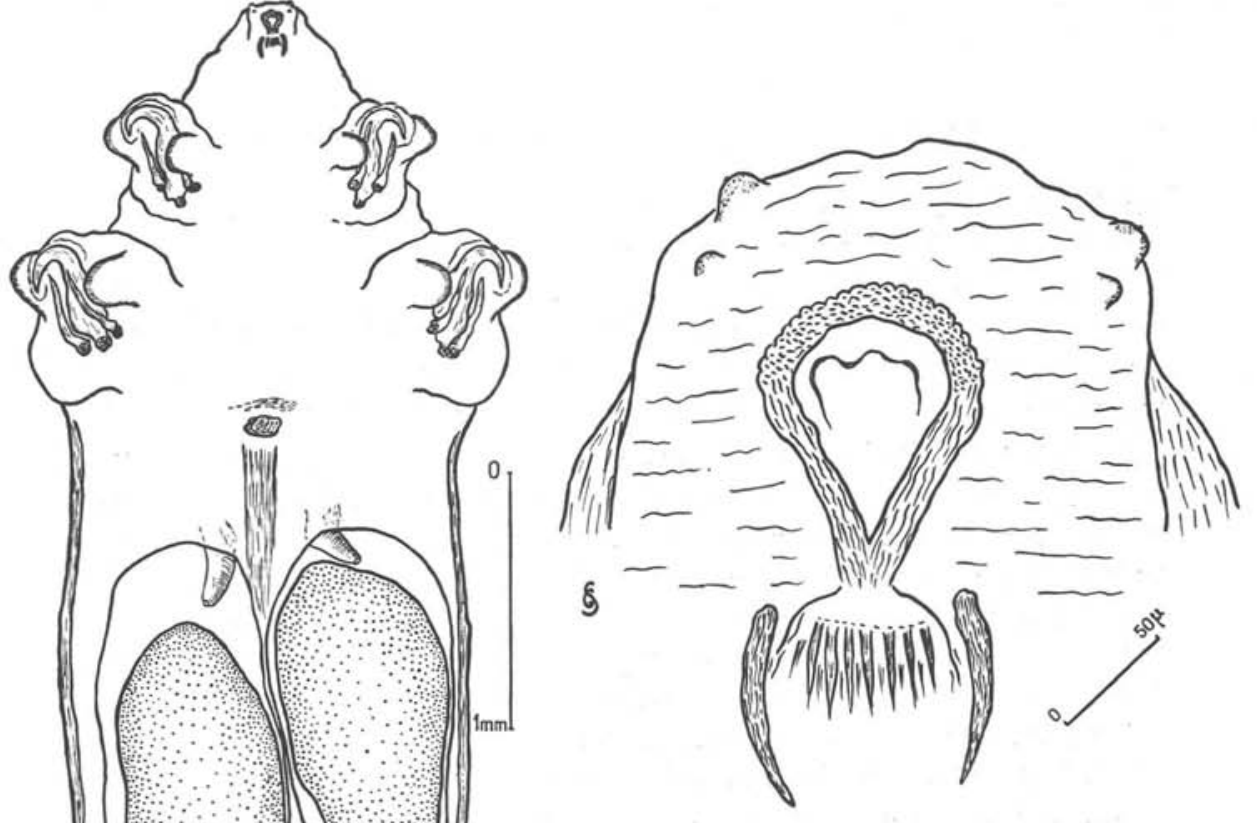

Fig. 12. - Complexe buccal de la femelle de

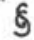
$R$. ampanihyensis

Fig. 11. - R. ampanihyensis, Céphalothorax de la femelle, avec partie abdominale antérieure (ovaires et oviductes vus après éclaircissement des téguments)

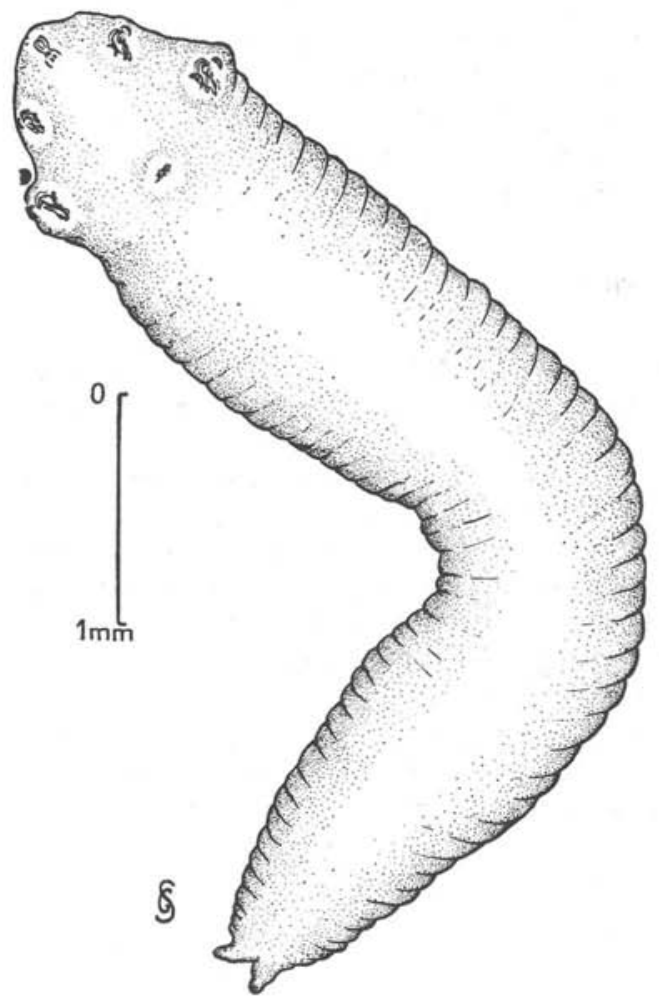

F1G. 13. - Mahafaliella n. gen. tetrapoda n. sp. (mâle) 
terminaux, les branches de la fourche caudale ainsi formée dépassant cependant largement l'extrémité postérieure de l'abdomen (fig. 10).

Par transparence on aperçoit au travers de la femelle, qui a une coloration jaune légèrement ambrée une fois fixée dans l'alcool, un tube intestinal ventral, peu circonvolutionné, de couleur marron foncé, qui, partant du cadre buccal, se termine au niveau des derniers anneaux abdominaux par un anus ventral.

Céphalothorax (fig. 11): Il a sensiblement la même forme que chez le mâle avec un complexe buccal cordiforme placé très en avant des deux paires de crochets supportés par des expansions cuticulaires bien développées dépassant latéralement le contour dorsal du céphalothorax.

Crochets antérieurs: Ils ont sensiblement la même forme que chez le mâle et leur partie antérieure crochue est engainée dans un repli du bord antérieur de l'expansion cuticulaire qui supporte le complexe crochet-parapodiales.

Longueur : $350 \mu$; épaisseur maximum : $125 \mu$.

Crochets postérieurs: longueur : $480 \mu$; épaisseur maximum : $150 \mu$.

Appareil génital (fig. 11): Le pore génital est situé ventralement juste en avant du premier anneau abdominal, à la limite céphalothorax-abdomen, immédiatement en arrière d'une ligne passant derrière les parapodes postérieurs.

Le vagin est court, rectiligne, et mesure environ $1 \mathrm{~mm}$. de long.

L'utérus est un long tube bourré d'œufs occupant la totalité de l'abdomen. Il est dorsal.

Les deux ovaires sont placés antérieurement à $500 \mu$ environ en arrière du pore génital. Ils sont oblongs et mesurent $1.250 \mu$ de long sur $685 \mu$ d'épaisseur environ. Ils sont enveloppés chacun d'une capsule à parois minces d'où part un oviducte musculeux qui débouche dans l'utérus (fig. 11).

Les œufs mesurent « in utero $\$ 125 \mu$ de long sur $85 \mu$ de large.

\section{Discussion}

Dans leur travail de 1936, Heymons et Vitzthum divisent les Raillietiella en cinq groupes qui sont les suivants: groupe geckonis, groupe kochi, groupe orientalis, groupe mediterranea et groupe furcocerca.

Le groupe geckonis est caractérisé par des espèces petites et d'allure trapue, dont les crochets postérieurs sont placés à l'extrémité d'un parapode très développé.

Les espèces du groupe kochi ont des appendices terminaux épais et charnus. Chez R. kochi Heymons, 1926, ils sont très longs et terminés par une portion cylindrö̈de. Le corps de la femelle présente un très fort étranglement en arrière du céphalothorax. La deuxième espèce du groupe, qui est $R$. shipleyi Heymons, 1926, a des appendices terminaux présentant un " bouton terminal ».

Dans le groupe orientalis, ces appendices sont grossiers et en forme de lèvres très épaisses, alors qu'ils sont très petits et arrondis dans le groupe mediterranea.

Chez les Raillietiella du groupe furcocerca, les appendices terminaux sont par contre très développés. 
L'espèce que nous venons de décrire appartiendrait donc à ce dernier groupe qui, à notre connaissance, comprend les espèces :

Raillietiella furcocerca (Diesing, 1836) Sambon, 1910.

Raillietiella giglioli Hett, 1924.

Raillietiella bicaudata Heymons et Vitzthum, 1936.

Raillietiella chamaeleonis Grétillat et Brygoo, 1959.

$R$. furcocerca est une très grande espèce $\left(\sigma^{*}=4 \mathrm{~cm}\right.$. $q=6 \mathrm{~cm}$.), avec des appendices terminaux trilobés (selon les figures de Heymons et Vitzthum, 1936). Il existe d'autre part un rétrécissement très marqué au niveau de l'union du céphalothorax et de l'abdomen.

D’après Miss Hett (1924), $R$. giglioli se différencie de l'espèce précédente par les caractères suivants: * (1) presence of two small papillae above the oral shield, (2) size, viz., length ; $26 \mathrm{~mm}$., breadth : $1 \mathrm{~mm}$. ». Et l'auteur conclut : « I therefore suggest that the name Raillietiella furcocerca should be retained for the larger species and that the species from Amphisbaena alba should be renamed $R$. giglioli n. sp. ».

Sans considérer, comme le font Heymons et Vitzthum (1936), R. giglioli comme synonyme de $R$. furcocerca, nous pensons que ces deux espèces sont très proches l'une de l'autre.

Chez $R$. bicaudata, la femelle mesure $43 \mathrm{~mm}$. environ et le mâle $11 \mathrm{~mm}$. Il y a 40 anneaux abdominaux dans les deux sexes, les appendices terminaux sont ventraux et non visibles dorsalement.

Les mâles et les femelles de $R$. chamaeleonis ont de 20 à 30 anneaux abdominaux. Les crochets antérieurs et postérieurs ont leur base formée par un « manche » bien développé. Les dilatateurs de copulation sont massués, leur extrémité antérieure étant terminée par un crochet, alors que leur partie moyenne est fortement chitinisée.

L'espèce que nous venons de décrire ne pouvant être rattachée à aucune des précédentes, nous la considérons comme nouvelle et l'appellerons Raillietiella ampanihyensis $\mathrm{n}$. sp., puisqu'elle a été trouvée pour la première fois chez un serpent capturé dans le District d'Ampanihy (Sud de Madagascar).

Holotype : Un mâle déposé dans les collections du Laboratoire de Zoologie des Vers au Muséum d'Histoire Naturelle de Paris ( $P^{r}$ A.-G. Chabaud).

Allotype : Une femelle déposée au même endroit.

Paratypes: Un mâle et une femelle déposés dans les collections de l'Institut Pasteur de Tananarive (Madagascar).

Un mâle et une femelle déposés dans les collections du Laboratoire National de Recherches Vétérinaires de Dakar (Sénégal).

Deux femelles dans les collections des auteurs.

Hôtes : Madagascarophis colubrina (Schlegel, 1837) (Ophidien) et Lioheterodon madagascariensis Duméril et Bribon, 1854 (Ophidien).

Localisation : Sacs aériens. 
Provenance : Tongobory et Vohitsara (district d'Ampanihy, Sud de Madagascar).

Dates : $25-4-1961$ et 4-4-1961.

Remarque : Considérant les difficultés que l'on rencontre dans la diagnose des différentes espèces de Raillietiella, en se référant à des caractères aussi fluctuants et peu précis que la forme et la longueur des appendices caudaux, nous pensons que la morphologie et les dimensions des crochets, des parapodiales et surtout des « dilatateurs de copulation » pourraient fournir des éléments facilitant considérablement l'étude taxonomique de ce groupe.

\section{Mahafaliella n. gen.}

Définition : Cephalobaenidae avec céphalothorax de contour semi-circulaire. Cadre buccal cordiforme. Papilles frontales et dorsolatérales très rapprochées et en position antérieure.

Les crochets disposés par paire forment un trapèze en arrière du cadre buccal. Chaque crochet est recouvert par un onychium et placé dans une petite cavité encadrée par deux lobiparapodiales et deux lèvres semi-circulaires.

Existence d'un crochet satellite bien développé, mais sans partie basale, placé ventralement par rapport à chaque crochet principal.

Crochets antérieurs plus petits que les postérieurs.

Pore génital mâle placé en position antérieure, juste en arrière du céphalothorax.

Abdomen segmenté terminé par deux appendices caudaux.

Espèce type:

\section{Mahafaliella tetrapoda n. gen., n. sp.}

Matériel : Ne comporte malheureusement qu'un seul exemplaire mâle récolté dans les sacs aériens d'un Acrantophis dumerili Jan, 1860 à Egogy, District d'Ampanihy (Sud de Madagascar), le 22-3-1961 (R. 94, 73/61).

\section{Description :}

Male (fig. 13).

Dimensions et habitus : Aspect de cigare, de couleur jaune ambré, après fixation dans l'alcool.

Longueur : 5,5 mm., largeur maximum : 1,1 mm. (vers le quart antérieur du corps).

Le céphalothorax, ou tête, a un contour semi-circulaire et ne présente pas de séparation bien marquée avec l'abdomen, qui est cylindrique et se rétrécit brusquement dans sa partie postérieure où il se termine par deux appendices 


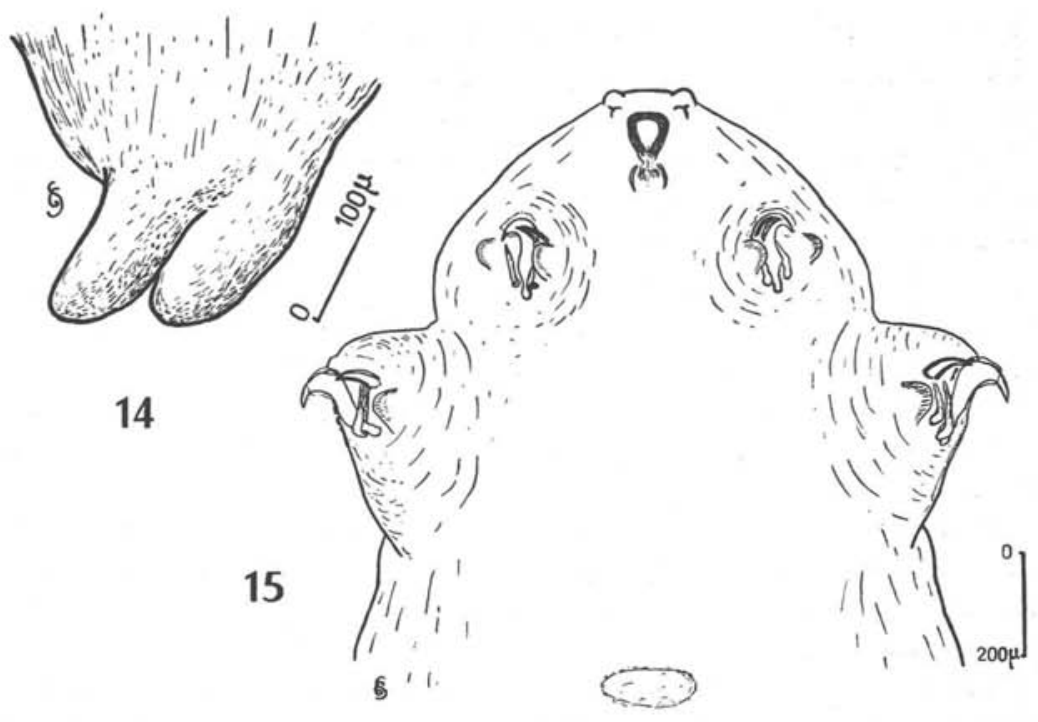

Fig. 14. - Mahafaliella n. gen. tetrapoda n. sp., appendices terminaux du mâle Fic. 15. - Céphalothorax du mâle de Mahafaliella tetrapoda

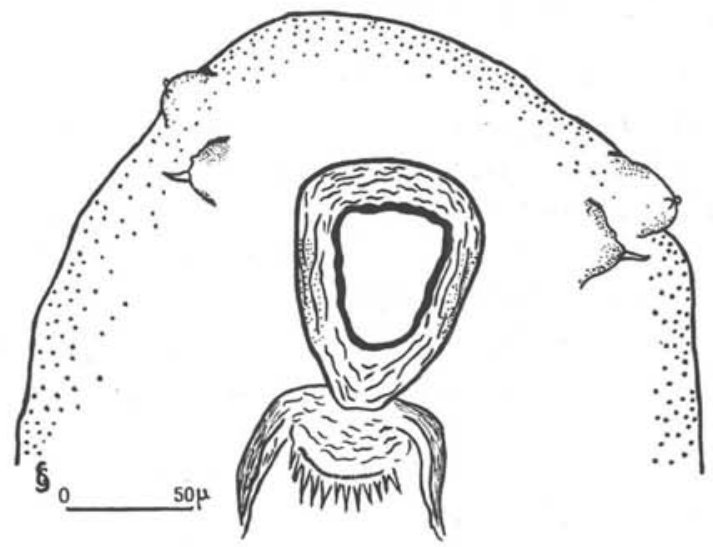

FIG. 16. - Cadre buccal et papilles céphaliques du mâle de $M$. tetrapoda 
charnus de $150 \mu$ de long sur $85 \mu$ d'épaisseur maximum à la base, mais sans * bouton terminal $\gg$ (fig. 14).

L'abdomen présente une segmentation discrète (42 anneaux).

Par transparence, on aperçoit le tube digestif non circonvolutionné, marron foncé, se terminant au niveau de la partie postérieure de l'abdomen.

Vers le quart antérieur du corps, la finesse de la cuticule, qui est très légèrement chagrinée, laisse apparaître une énorme vésicule séminale ovalaire, allongée d'avant en arrière et de couleur foncée.

Céphalothorax: Sur son bord antérieur sont placées deux papilles dorsolatérales bien développées et arrondies et immédiatement en arrière deux papilles frontales terminées chacune par un prolongement ayant l'allure d'une soie sensorielle, d'ailleurs très difficilement visible (fig. 16).

Le cadre buccal, cordiforme et très chitinisé, est placé immédiatement en arrière des papilles.

Les crochets, disposés par paires, forment un ensemble trapézoïdal et sont placés au sommet de quatre parapodes dont les postérieurs, les plus développés, débordent largement le contour externe de la tête quand ils sont en complète extension (fig. 15).

Chaque crochet, dont la base est rétrécie en forme de « manche », est placé dans une petite cavité encadrée par deux lobiparapodiales faiblement chitinisées flanquées latéralement de deux lèvres semi-circulaires à contour plus ou moins distinct (fig. 17). La partie supérieure de chaque crochet est coiffée par un onychium à paroi mince et finement plissée, qui s'insère sur le bord supérieur de la cavité uncéale. Immédiatement contre le bord supérieur latéro-ventral de cette cavité et fixé à une embase musculaire, existe un crochet satellite bien développé, mais sans renflement basal.

Dimensions des crochets (fig. 18 et 19):

Crochet antérieur: longueur: $150 \mu$; épaisseur maximum : $35 \mu$.

Lobiparapodiales antérieures: longueur : $100 \mu$.

Crochet satellite antérieur : longueur : $83 \mu$; largeur à la base : $40 \mu$.

Crochet postérieur : longueur : $187 \mu$; épaisseur maximum : $45 \mu$.

Lobiparapodiales postérieures : longueur : $115 \mu$.

Crochet satellite postérieur : longueur : $105 \mu$; largeur à la base : $40 \mu$.

Appareil génital: Les deux testicules, qui mesurent environ $310 \mu$ de long sur $170 \mu$ d'épaisseur, sont ovoïdes et placés latéralement le long de la paroi abdominale, au niveau des six à sept premiers anneaux abdominaux. La vésicule séminale est ovalaire (longueur : $550 \mu$; largeur : $300 \mu$ ) et allongée ventralement entre les deux testicules. Les deux canaux déférents qui en partent sont circonvolutionnés et présentent sur leur parcours une série de renflements ampoulaires avant de s'accoler à la face interne des * dilatateurs de copulation $>$. 


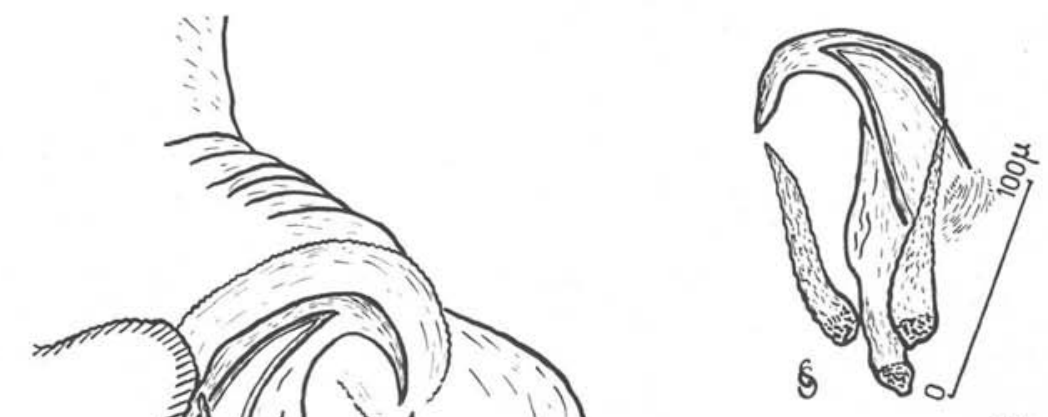

18
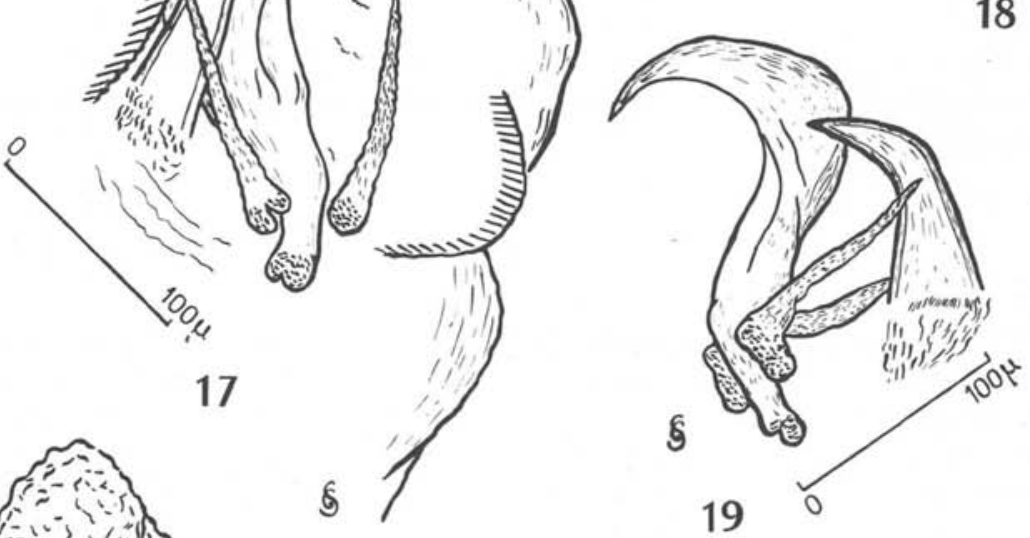

FIG. 17. - Complexe parapodial du mâle de $M$. tetrapoda

FIG. 18. - Crochet antérieur et parapodiales du mâle de $M$. tetrapoda

Fig. 19. - Crochet postérieur et parapodiales du mâle de $M$. tetrapoda

Fig. 20. - Mahafaliella n. gen. tetrapoda n. sp. A) \& Dilatateur de copulation ». B) Détails de l'ornementation de la tête des « dilatateurs de copulation 》

20 
Chaque * dilatateur , (fig. $20 \mathrm{~A}$ ) est formé de deux parties distinctes:

Une partie postérieure en forme de massue dont la tête est finement striée (détails des striations, fig. $20 \mathrm{~B}$ ) et le manche terminé par de petites digitations. Cette partie mesure $500 \mu$ de long.

Une partie antérieure styloïde, de $450 \mu$ de longueur, légèrement recourbée en $S$, fortement chitinisée et s'attachant postérieurement par un faisceau musculaire à la tête de la partie postérieure du dilatateur. Elle se termine en avant par un onglet articulé de $145 \mu$ de long dont la pointe est coiffée de deux longs fouets.

Les deux canaux éjaculateurs convergent vers le pore génital au sommet d'une petite élevure cutanée, juste en arrière du céphalothorax, sur le premier segment abdominal.

\section{Discussion}

L'espèce que nous venons de décrire présente certains caractères généraux du genre Raillietiella, tels que la disposition trapézoïdale des parapodes en arrière du cadre buccal, l'existence de parapodiales, la présence de deux appendices caudaux terminaux, la position antérieure du pore génital. Elle ne peut cependant être rattachée à ce genre en raison de la présence d'un crochet satellite sur chaque parapode.

Nous ne pouvons pas non plus l'inclure dans le genre Cephalobaena Heymons, 1922, où il n'y a pas de crochet satellite et où les crochets antérieurs et postérieurs sont de mêmes dimensions.

Dans le genre Megadrepanoides Self et Kuntz, 1957, il existe bien un crochet auxiliaire au niveau des parapodes postérieures, mais les crochets antérieurs sont bien plus grands que les postérieurs, qui sont en outre pourvus d'un fulcrum.

En conséquence, nous rangeons notre espèce dans le genre Mahafaliella $\mathrm{n}$. gen., ainsi nommé afin de rappeler que ce Pentastome a été trouvé chez un Ophidien du pays Mahafaly (Sud de Madagascar) et nous l'appellerons Mahafaliella tetrapoda n. gen., n. sp. puisque chez elle les parapodes sont bien développées.

Holotype : Un mâle, déposé dans les collections du Muséum d'Histoire Naturelle de Paris, Laboratoire de Zoologie des Vers ( $\mathrm{P}^{r}$ Chabaud).

Femelle : Inconnue.

Hôte : Acrantophis dumerili Jan, 1860 (Ophidien).

Localisation: Sacs aériens.

Provenance : Egogy, district d'Ampanihy (Sud de Madagascar). Date : 22-3-1961. 


\section{Clé des genres de la famille des CEPHALOBAENIDAE Heymons}

1) Crochets antérieurs plus petits que les crochets postérieurs ...... 4

2) Crochets antérieurs de mêmes dimensions que les crochets postérieurs, qui sont tous placés à l'extrémité de parapodes pouvant atteindre en complète extension la longueur de la tête ........ Cephalobaena Heymons, 1922

3) Crochets antérieurs beaucoup plus grands que les crochets postérieurs. Présence d'un crochet satellite sur les parapodes postérieurs . . . . . . . .

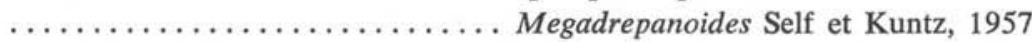

4) Absence de crochet satellite sur chaque parapode Raillietiella Sambon, 1910

5) Présence d'un crochet satellite sur chaque parapode . Mahafaliella n. gen.

\section{Remarque :}

Dans la clé ci-dessus nous avons inclu le genre Megadrepanoides Self et Kuntz, 1957, mais nous pensons que ce genre n'appartient pas à la famille des Cephalobaenidae ni à l'ordre des Cephalobaenida.

En effet, Self et Kuntz en 1957, dans leur description de l'espèce type qui est Megadrepanoides salomonensis, ainsi que dans celle de $M$. varani Self et Kuntz, 1957, signalent la présence d'un fulcrum, * Length of fulcrum equal to that of hooks », à la base des crochets postérieurs. Or, si l'on se réfère à la définition de l'ordre des Cephalobaenida par Heymons et Vitzthum en 1936, les crochets des Pentastomes de cet ordre ont une forte protection chitineuse, mais pas de fulcrum; d'autre part, leur cadre buccal est placé en avant des crochets antérieurs.

* Mundöffnung vor den Haken gelegen »... * Die Haken sitzen in Vertiefungen oder flachen Gruben und besitzen höchstens chitinöse Stützleisten, aber kein basales Stützgerüst (Fulcrum). ».

Comme d'autre part, chez Megadrepanoides, les lobiparapodiales existent, tout au moins au niveau des crochets antérieurs du mâle, et que les femelles ont un orifice génital placé antérieurement, ce genre n'appartient pas non plus à l'ordre des Porocephalida Heymons et Vitzthum 1936, où les parapodiales sont absentes et où l'orifice génital est postérieur.

Pour des raisons de commodité, il y a lieu cependant de maintenir ce genre dans l'ordre des Cephalobaenida et la famille des Cephalobaenidae, en attendant une révision générale des Pentastomides.

\section{Formes immatures de Raillietiella sp.}

Le prélèvement R. $145, \mathrm{n}^{\circ} 121 / 61$, provenant des sacs aériens d'un Madagascarophis sp. (Ophidien) capturé à Manombo, District d'Ambovombe (Sud de Madagascar), le 26-7-1961, comportait quatre femelles impubères de Raillietiella sp., présentant des œufs non embryonnés dans leur utérus. 
Quant au prélèvement R. $148, \mathrm{n}^{\circ} 124 / 61$, il ne comportait qu'un jeune mâle de Raillietiella sp. récolté dans la trachée d'un Mimophis mahafalensis (Grandidier, 1867), capturé à Zampongotra, District d'Ambovombe (Sud de Madagascar), le 27-7-1961.

\section{Formes larvaires de Raillietiella sp.}

Nous donnons ci-dessous la description de formes larvaires de Pentastomes récoltées à l'autopsie d'un Chamaeleo oustaleti Mocquard, 1894, capturé à Ampijoroa (Tsaramandroso, Madagascar), le 10-11-1961 (C. 217, $\mathrm{n}^{\circ}$ 196/61).

Le matériel comporte neuf larves recueillies dans les sacs aériens de ce caméléon.

De par la disposition et la forme de leurs crochets, et la position du cadre buccal placé en avant du trapèze parapodial, nous rapportons ces larves au genre Raillietiella.

\section{Description :}

La larve qui a un aspect trapu mesure environ $100 \mu$ de long, pour une largeur maximum de $500 \mu$, au niveau des crochets postérieurs (fig. 21).

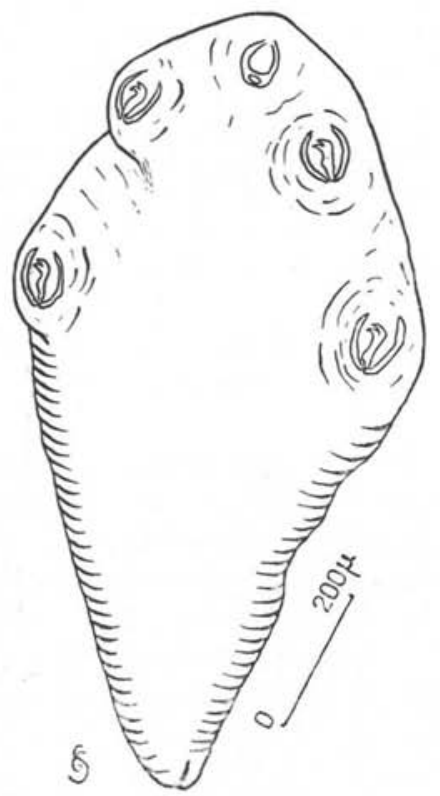

Le céphalothorax occupe la moitié du corps et porte en avant, sur sa face ventrale, un cadre buccal de forme ovalaire fortement chitinisé.

Les quatre crochets sont disposés par paires, chacun d'eux étant placé dans une petite cavité située au sommet d'une petite élevure peu saillante mais cependant bien marquée (parapode).

FIG. 21. - Larve de Raillietiella sp.

Les crochets, qui sont fortement chitinisés, ont une base cylindrique ou * manche » qui se poursuit en avant par une portion plus massive terminée par deux pointes recourbées (fig. 22). 
Encadrant chaque crochet, et posées sur les marges de la cavité où il est logé, existent deux baguettes très chitinisées, concaves vers l'intérieur, à l'extrémité antérieure flabelliforme, et dont les bases sont réunies par un pont chitineux plus mince. Ces deux éléments semblent bien être, de par leur emplacement, leur forme et leur dimension, les futures lobiparapodiales de la nymphe et de l'adulte.
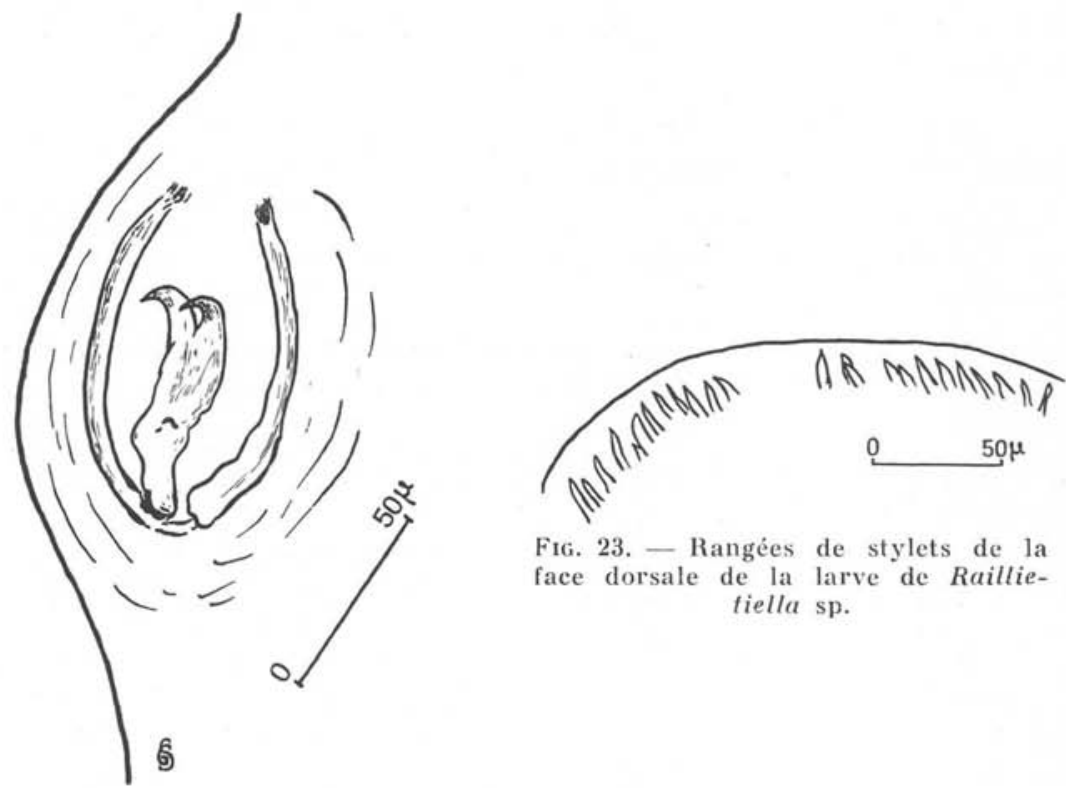

Fig. 23. - Rangées de stylets de la face dorsale de la larve de Railliefiella sp.

Fig. 22. - Complexe parapodial de la larve de Raillietiella sp.

Les crochets antérieurs et postérieurs ont sensiblement les mêmes dimensions (longueur : 55 à $60 \mu$; épaisseur maximum : 14 à $15 \mu$ ).

La face dorsale du céphalothorax présente sur son bord antérieur deux rangées de 12 petits stylets chitineux de 10 à $15 \mu$ de longueur, disposées de part et d'autre de l'axe longitudinal du corps de larve (fig. 23).

L'abdomen, qui est conique, se termine postérieurement par deux appendices caudaux terminaux à peine marqués et à allure de mamelons. Il existe une ébauche de segmentation abdominale (20 à 22 segments).

Il est bien entendu très malaisé d'attribuer un nom d'espèce à des formes larvaires de Raillietiella. Cependant, la forme générale des crochets, le nombre d'ébauches de segments abdominaux et le fait d'avoir été trouvées chez un Chameleo oustaleti, où le seul Raillietiella décrit jusqu'à ce jour est $R$. chame- 
leonis, nous laisse supposer qu'il s'agit peut-être des formes larvaires de cette espèce, sans toutefois pouvoir l'affirmer.

\section{RÉSUMÉ}

L'étude d'une petite collection de Pentastomes récoltés au cours de l'année 1961, à l'autopsie d'un certain nombre de reptiles à Madagascar, nous a permis de décrire :

$1^{\circ}$ La forme et les dimensions des « dilatateurs de copulation » dans l'espèce Gigliolella brumpti (Giglioli, 1922) Chabaud et Choquet, 1954.

$2^{\circ}$ Raillietiella ampanihyensis $\mathrm{n}$. sp. récolté dans les sacs aériens de Madagascarophis colubrina (Schlegel, 1837) et de Lioheterodon madagascariensis Duméril et Bribon, 1854.

$3^{\circ}$ Un nouveau genre de Cephalobaenidae, Mahafaliella $\mathrm{n}$. gen. avec comme espèce type Mahafaliella tetrapoda n. sp., malheureusement à l'aide d'un seul exemplaire mâle, récolté dans les sacs aériens d'un Acrantophis dumerili Jan, 1860 , et se différenciant des Raillietiella, par la présence d'un crochet auxiliaire sur chaque parapode.

$4^{\circ}$ Des formes larvaires de Pentastomes récoltées chez un Chamaeleo verrucosus Cuvier, 1829, que nous avons identifiées comme appartenant au genre Raillietiella, et peut-être à l'espèce $R$. chamaeleonis Grétillat et Brygoo, 1959 .

D'autre part, nous avons retrouvé Raillietiella chamaeleonis chez Lioheterodon modestus (Gunther, 1863), alors qu'il n'avait été décrit et signalé que chez les caméléons Chamaeleo oustaleti Mocquard, 1894 et C. verrucosus.

\section{BIBLIOGRAPHIE}

Bovien (P.), 1927. - Ueber einige Pentastomiden aus Java. Vidensk. Medd. fra Densk. Naturh. Forening, 84, pp. 1-9.

Chabaud (A.-G.) et Chopuet (M.-T.), 1954. - Nymphes du Pentastome, Gigliolella (n. gen.) brumpti (Giglioli, 1922), chez un Lémurien. Riv. Parasit. Ital., 15, pp. 331-336.

Gedoelst (L.), 1921. — Un linguatulide parasite d'un Batracien. Rec. Ind. Mus., p. 22.

Giglioli (G.), 1922. - Armillifer brumpti n. sp., nouvelle espèce de Linguatulidé de Madagascar. Bull. Soc. Path. Exot., 15, pp. 565-572.

- 1923. - On the linguatulid arachnid Raillietiella furcocerca. Proc. Zool. Soc. Lond., pp. 15-18.

GrÉtillat (S.) et Brygoo (E.-R.), 1959. - Raillietiella chamaeleonis n. sp., première espèce de Cephalobaenidae (Pentastomida) signalée à Madagascar. Ann. Parasit. Hum. Comp., 34, pp. 112-120. 
- - 1961. - Les \& dilatateurs de copulation » chez Raillietiella (Heymonsia) hemidactyli, et valeur taxonomique possible de ces organes chez les Pentastomida. Arch. Inst. Past. Madagascar, 29, pp. 71-74.

Hetr (M. L.), 1924. - On the family Linguatulidae. Proc. Zool. Soc. Lond., pp. 107-159.

- 1934. - On a collection of Linguatulids (Pentastomida) from Burma, with description of a new subgenus. Proceed. of the general meeting for scientific business of the Zoological Society of London, July 1934, part. 2, pp. $425-431$.

Heymons (R.) und Vitzthum (H. Graf), 1936. - Beiträge zur Systematik der Pentastomiden. Zeitschr. f. Parasitenk., 8, pp. 1-113.

- - 1939. - Beiträge zur Systematik der Pentastomiden. II. Einige Bemerkenswerte Pentastomiden aus Lacertilien. Zeitschr. f. Purasitenk., 10, pp. 675-690.

SAmbon (L. W.), 1922. - A synopsis of the family Linguatulidae. Jl. Trop Med. Hyg., 25, pp. 188-206.

Self Teague (J.) and Kuntz (R. E.), 1957. - Pentastomids from African reptiles and mammals, and from reptiles of Florida Islands, British Salomon Islands (South Pacific). Jl. Parasit., 43, pp. 194-200.

(Laboratoire National de Recherches Vétérinaires de Dakar, Sénégal et Institut Pasteur de Madagascar, Tananarive) 